\title{
Low Expression of STING Promoted Endometrial Stromal Cells Invasion and Migration Via STING/IRF3/IFNb1 Pathway in Endometriosis Eutopic Endometrium
}

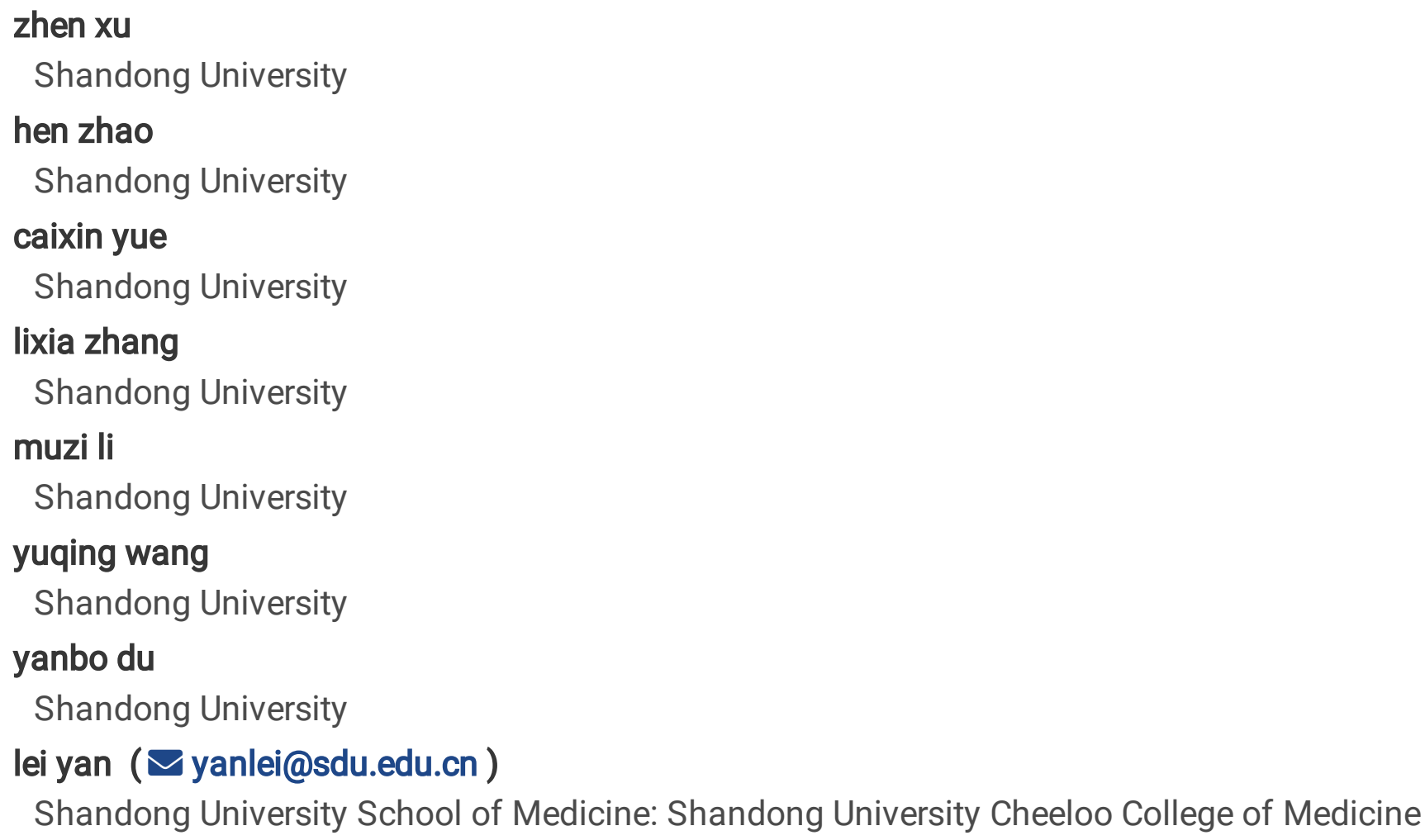

\section{Research}

Keywords: endometriosis, innate immunity, eutopic endometrium, invasion, migration

Posted Date: July 28th, 2021

DOl: https://doi.org/10.21203/rs.3.rs-709514/v1

License: (c) (i) This work is licensed under a Creative Commons Attribution 4.0 International License. Read Full License 


\section{Abstract}

Background: Recent studies have confirmed that endometriosis is a chronic inflammatory disease. In our previous work, we found that STING (stimulator of interferon genes) was differentially expressed in eutopic endometrium and controlled endometrium by proteomics.

Method: we used the 11 pairs of samples to verify STING expression by WB and IHC experiments. We detected cells proliferation by EdU assays, cells invasion and migration by Transwell assays. The effect of signaling pathway in HESC was detected by WB and Elisa expreriments.

Results: STING was significantly lower expressed in eutopic endometrium of endometriosis, while IHC results showed that STING was expressed in both stroma and glandular epithelium of normal endometrium, but in endometriosis, STING was mainly expressed in the stroma of eutopic endometrium, and mainly in glandular epithelium of ectopic endometrium. Further study on the role of STING on endometrial stromal cells showed that low expression of STING could promote the HESC proliferation by EdU experiments, invasion and migration by Transwell experiments. The effect of STING/IRF3/IFNb1 signaling pathway in HESC with low expression of STING was also reduced, mainly showed the decreased expression of phosphorylated IRF3 and TBK1, and the decreased secretion of IFNb1. In order to further study the effect of IFNb1, secreted by STING/ IRF3/IFNb1 signaling pathway, on stromal cells, we added exogenous IFNb1 to the HESC with low expression of STING, and found that IFNb1 could reverse the invasion and migration function of stromal cells, but little effect on cell proliferation.

Conclusions: We clarified that STING expressed mainly in stromal tissues and lower in endometriosis eutopic endometrium compared to normal endometrium. Low expressed STING promoted stromal cells invasion and migration via STING/IRF3/IFNb1 signaling pathway.

\section{Introduction}

Endometriosis is gernerally considered as a chronic inflammation disease, ectopic lesions of endometriosis formed by eutopic endometrium after immune escape, invasion and migration[1]. In addition, a meta-analysis showed that there was a correlation between endometriosis and autoimmune diseases[2]. The immune related pathogenic factors of endometriosis are mainly divided into the following two parts: the immune dysfunction of endometriosis and the imbalance of immune microenvironment in ectopic lesions [1, 3-6].

In our previous study[7], the endometrial samples were identified by proteomics technology, we found that the differential proteins expression of innate immune proteins in endometriosis accounted for more. After further analysis, we found that the expression of stimulator of interferon genes (STING) decreased in eutopic endometrium of endometriosis. STING, also known as TMEM173, as a key DNA receptor adaptor protein, plays an important role in the innate immune signal pathway induced by virus. Cyclic GMP-AMP synthase (cGAS)-STING signal pathway is also a key pathway in the innate immune process[8]. STING also plays different roles in many organ diseases, including lung cancer cells, synovial cells, breast 
cancer cells and so on[9-12]. One study reported that the expression of STING protein in ectopic endometrium with endometriosis was higher than that in eutopic endometrium[13], and the expression of STING protein in glandular epithelium was higher than that in stromal cells. However, this article did not compare the expression of STING protein in eutopic endometrium with endometriosis and normal endometrium without endometriosis, also did not explore the mechanism, which indicating that this field still needs further exploration.

We collected eutopic endometrium and ectopic endometrium with endometriosis and eutopic endometrium of normal women for immunohistochemical analysis, which based on proteomics research. It was found that STING protein was only expressed in stromal tissue of eutopic endometrium with endometriosis, which was lower than that of normal women. Low expression of STING protein promoted the proliferation of endometrial stromal cells invasion and migration function.

\section{Materials And Methods}

\section{Patients and sample collection}

Fresh endometrial eutopic tissues from 11 patients with endometriosis were collected during the late proliferative phase of the menstrual cycle (Day11 13) and from 11 women of reproductive age who underwent oviduct obstruction as controls. The diagnosis was confirmed by laparoscopy. Samples were collected from October 2018 to December 2019 at the Reproductive Hospital Affiliated to Shandong University. Women undergoing hysteroscope-laparoscopic surgery for suspected endometriosis were confirmed by histological examination, no comorbidities such as PCOS, POI or IBD, so did the controls. Informed consent was obtained from patients before surgery. All surgical patients received no hormonal treatments 3 months prior to surgery. Patients data were collected, including age, body mass index, and hormone levels. The study was approved by the Institutional Review Board (IRB) of the Reproductive Hospital Affiliated to Shandong University.

Cell culture and transfection assay

Human endometrial stromal cells (HESC) were cultured with 90\% non-phenol red DMEM/F12 (Gibco) supplemented with $10 \% \mathrm{FBS}$ and $1 \% \mathrm{P} / \mathrm{S}$ in a $5 \% \mathrm{CO} 2$ atmosphere at $37^{\circ} \mathrm{C}$. si-STING were purchased from Genepharma (GenePharma, Co., Ltd., Shanghai, China). When HESC reached $70-80 \%$ confluence, transfection was conducted with Lipofectamine 3000 (Invitrogen, Carlsbad, CA, USA) according to the manufacturer's instructions. Cells were harvested after $48 \mathrm{~h}$ for other assays.

Protein extraction and western blotting

Radioimmunoprecipitation assay buffer (RIPA, Beyotime, Shanghai, China) was used to lyse cells after transfection for $48 \mathrm{~h}$, followed by the determination of the protein concentration using bicinchoninic acid (BCA; Beyotime). The protein was separated by $10 \%$ sodium dodecylsulphate-polyacrylamide gel electrophoresis (SDS-PAGE), followed by transfer to a polyvinylidene difluoride (PVDF) membrane 
(Immobilon-P). After blocking in 5\% non-fat milk at room temperature for $1 \mathrm{~h}$, the primary antibody was added and incubated in a refrigerator at $4^{\circ} \mathrm{C}$ overnight. Tris-buffered saline-Tween (TBST) was used to wash the membrane for 15 min 3 times, and the corresponding secondary antibody was added and incubated for $1 \mathrm{~h}$ at room temperature (about $25^{\circ} \mathrm{C}$ ). After washing each sample for $15 \mathrm{~min} 3$ times with TBST, the protein was detected using the ECL detection system (Millipore, Billerica, MA, USA). Primary antibodies against rabbit anti-human STING (1:1000 dilution, CST13647, USA), rabbit anti-human TBK1(1:1000 dilution, CST38066, USA), rabbit anti-human p-TBK1(1:1000 dilution, CST5483, USA), rabbit anti-human IRF3(1:1000 dilution, CST4302, USA), rabbit anti-human p-IRF3(1:1000 dilution, CST29047, USA), and mouse anti-human Tublin (1:10000 dilution; Proteintech 11224-1-Ig, Rosemont, IL, USA) were used. Goat anti-rabbit and Goat anti-mouse HRP-conjugated secondary antibodies were obtained from ZSGB-BIO (1:10000 dilution, Beijing, China).

5-Ethyl-2'-Deoxyuridine Incorporation (EdU) assay

For the EdU assay, HESC were incubated with $50 \mu \mathrm{mol} / \mathrm{L}$ EdU (RiboBio, Guangzhou, China) for $6 \mathrm{~h}$ at $37^{\circ} \mathrm{C}$ and then fixed with $4 \%$ paraformaldehyde for $30 \mathrm{~min}$ at room temperature. After incubation with Apollo reaction reagent for $30 \mathrm{~min}$, the cells were stained with Hoechst 33342. The Olympus 1X51 (Tokyo, Japan) was used to capture images of HESC, and cells in five random fields per group were counted. The EdU incorporation rate was estimated as the ratio of EdU-positive cells to Hoechst-positive cells.

Transwell assays

Transwell assays were performed using 24-well plates with 8- $\mu \mathrm{m}$ pore size inserts (Corning Life Sciences, Corning, NY, USA) according to the manufacturer's protocols. HESC were plated into Transwell chambers after $48 \mathrm{~h}$ of transfection. The essential difference between the migration and invasion assays is that, for migration assays cells can migrate through the inserts directly, but for invasion assays they have to invade the matrix to reach the inserts. Details are shown as follows.

In the migration assay, the HESC $\left(10^{5}\right.$ cells/well $)$ were seeded into the upper chamber in $200 \mu \mathrm{L}$ of serumfree phenol red-free DMEM/F12 and allowed to migrate for $24 \mathrm{~h}$ to the lower chamber, which contained phenol red-free DMEM/F12 with $10 \%$ FBS.

In the invasion assay, Matrigel ( $1 \mathrm{mg} / \mathrm{mL}$; BD Biosciences, Franklin Lakes, NJ, USA), prepared in serumfree phenol red-free DMEM/F12 medium, was placed in the upper chamber and incubated for $8 \mathrm{~h}$ at $37^{\circ} \mathrm{C}$. Similar to the migration assay, HESC $\left(10^{5}\right.$ cells/well) were seeded in the upper chamber with $200 \mu \mathrm{L}$ of serum-free medium and allowed to invade the lower chamber, which contained medium with $10 \%$ FBS, for $36 \mathrm{~h}$.

Transwell filters were fixed with $4 \%$ paraformaldehyde for 30 min, stained with hematoxylin for 30 min, and fixed on a glass slide. Cells that did not migrate or invade were wiped off with cotton swabs. The results were expressed as the number of cells that migrated or invaded per field, as counted using the Olympus 1X51 microscope in five random fields. 
Immunohistochemistry analysis

Fresh tissue samples were washed with PBS several times to remove blood. Then, they were fixed in $4 \%$ PFA for $24 \mathrm{~h}$ and embedded into paraffin. The samples were cut into 4 um sections and mounted onto glass slides. Deparaffinized, rehydrated sections were incubated with $3 \% \mathrm{H}_{2} \mathrm{O}_{2}$ for $30 \mathrm{~min}$ to block endogenous peroxidase activity. Antigen retrieval was performed using a pressure-cooker for $15 \mathrm{~min}$ in EDTA buffer in boiling water. The sections were rinsed in PBS, blocked with $10 \%$ BSA for $30 \mathrm{~min}$, and then incubated with primary antibodies rabbit anti-human STING (diluted 1:100, CST13647, USA) overnight in a wet chamber at $4{ }^{\circ} \mathrm{C}$. HRP-conjugated goat anti-rabbit or mouse IgG was used as the second antibody. HRP activity was detected by measuring the level of the DAB for several seconds. The sections were counterstained with haematoxylin before mounting. The sections were observed under an Olympus 1 X51 microscope.

\section{Elisa experiment}

For the Elisa assay, protocols were based on Human IFN-beta ELISA Kit (R\&D 41410-1). Add 50ul sample diluent, 50ul standard in blank microtiter plate, incubated $1 \mathrm{~h}$ then aspirated and wash 3 times. Add 100ul diluted HRP solution, incubated $1 \mathrm{~h}$ then as aspirated and wash 3 times. Add 100ul TMB substrate, incubated $15 \mathrm{~min}$ in the dark do not seal or wash. Add 100ul Stop Solution and read plate within $5 \mathrm{~min}$ at $450 \mathrm{~nm}$.

\section{Statistical Analysis}

Statistical analyses were performed using SPSS 21.0 (Statistical Package for the Social Sciences, SPSS, Inc., Chicago, IL, USA). Values were expressed as means \pm SD, and comparisons were performed using Student's t-tests. $P<0.05$ was considered statistically significant. Each experiment was repeated three times.

\section{Results}

Analysis of proteomics results suggested that NF-kB and AP-1 pathways play an important role in inflammation related signaling pathways, and these two pathways are downstream of STING (Supplemental figure).

\section{Expression of STING in endometriosis}

WB results showed that the expression of STING significantly decreased in eutopic endometrium with endometriosis compared to the controls (Fig. 1-A). By IHC images (Fig. 1-B), we analyzed eutopic endometrium, ectopic endometrium with endometriosis and normal control endometrium, found that STING was expressed in stroma and glandular epithelium of normal control endometrium, but in eutopic endometrium with endometriosis, STING was significantly decreased compared to control endometrium, 
and mainly expressed in stroma in eutopic endometrium however epithelial tissue in ectopic endometrium.

STING was essential for HESC function

As we found that STING expressed decreased in eutopic endometrium with endometriosis and mainly in stroma, we used HESC knockdown STING to explore influence of STING on HESC function, in order to verify STING function on endometriosis. When HESC knockdown STING (transfected with si-STING), EdU assay manifested that HESC proliferation was increased significantly compared to NC (transfected with negative control), si-STING HESC compared to NC group was $0.2866 \pm 0.01470$ vs. $0.6911 \pm 0.01796$, ${ }^{* \star \star \star} \mathrm{P}<0.0001$ (Fig. 2-A); Transwell assay showed that HESC invasion(130.0 \pm 6.296 vs. $424.1 \pm 22.31$, $\left.{ }^{\star \star \star \star} \mathrm{P}<0.0001\right)$ and migration( $82.93 \pm 6.940$ vs. $\left.82.93 \pm 6.940,{ }^{\star \star \star \star} \mathrm{P}<0.0001\right)$ were increased significantly after STING knockdown compared to NC (Fig. 2-B).

STING/IRF3/IFNb1 pathway in HESC

To explore that how did STING play an important role in HESC, we knockdown STING and study the protein levels of downstream IRF3, pho-IRF3, TBK1, pho-TBK1 and IFNb1, those are STING pathway proteins. Western blot results showed that pho-IRF3 and pho-TBK1 were decreased in STING knockdown group compare to controls, leaded to IFNb1 decreased finally, whish showed in Elisa assay(Fig. 3-B). We aimed at that how did IFNb1 influence HESC function, then we added exogenous IFNb1 in HESC with knockdown STING (siSTING) and NC, found that invasion and migration of HESC were decreased reversely, HESC invasion (IFNb1-NC and IFNb1-siSTING $274.7 \pm 7.767$ vs. $135.7 \pm 12.63,{ }^{* \star *} \mathrm{P}<0.0001$ ) and migration (IFNb1-NC and IFNb1-siSTING $28.53 \pm 3.625$ vs. $28.53 \pm 3.625,{ }^{* \star *} \mathrm{P}<0.0001$ ) decreased in siSTING with IFNb1 compared to siSTING, but could not completely reverse the invasion (siSTING and IFNb-siSTING $222.9 \pm 10.16$ vs. $124.1 \pm 11.77,{ }^{\star \star \star} \mathrm{P}<0.0001$ ) and migration (siSTING and IFNb-siSTING $49.13 \pm 4.241$ vs. $135.7 \pm 12.63, \star \star \star \star P<0.0001)$. However exogenous IFNb1 in HESC with knockdown STING showed no influence on HESC proliferation, cells proliferation was not decreased in siSTING with IFNb1 compared to siSTING (siSTING and IFNb-siSTING $0.6874 \pm 0.02081,0.7187 \pm 0.02638, P=0.795$ ) (Fig. 3-C,D).

\section{Discussion}

In this study, we started with proteomic sequencing of endometriosis eutopic endometrium and normal endometrium, and found that significant difference of proteins related to immune factors in endometriosis group. According to further analysis, we focused on innate immune protein STING. By verifying expression of STING in endometriosis eutopic endometrium, we found that STING was expressed lower in endometriosis compared to controls. The expression of STING in eutopic endometrium is mainly in the endometrial stroma, and the decreased of STING lead to increase the proliferation, invasion and migration of endometrial stromal cells. Further study of STING protein signaling pathway showed that the phosphorylation of STING/IRF3/IFNb1 also decreased in the HESC 
when STING expression was knockdown, which means that STING signaling pathway was inhibited. By exogenous adding IFNb1, the invasion and migration of stromal cells were reversely decreased, which indicated that STING signaling pathway affected the function of endometrial stromal cells.

At present, it is believed that environment will also affect the molecular structure and function of endometriosis endometrium, which resulted in formation of ectopic lesions, and the imbalance of the immune environment of endometriosis is an important reason for the formation of ectopic lesions. The imbalances of peripheral circulating immune system and female intrauterine endometrium autoimmunity, those can lead to the occurrence of ectopic lessions of endometriosis, which is associated with infertility, early abortion and abnormal histometaplasia[4, 14, 15]. One transcriptome analysis of endometrium with endometriosis found that signaling pathways including lymphocyte activation, antigen presentation, cytokine induction and inflammation were up-regulated, those were related to the changes of steroid hormone expression. This result was similar to our proteomic analysis[16, 17]. We found that signaling pathways related to immune factors were changed in eutopic endometrium with endometriosis. Gene analysis of endometrium tissue of endometriosis showed that the immune and inflammatory factors of progesterone decidualization were important factors affecting endometriosis endometrial receptivity and maintaining pregnancy[18]. Although it is not clear that the cellular components of endometrial tissue are causes of pro-inflammatory and pro-immune, still need more researches to confirmed, especially, immuned cells migrated and fibrotic cells of endometrial matrix in eutopic endometrium[14, 19, 20]. In conclusion, recent studies have shown that environment of pro-inflammatory in endometriosis is an important reason for the formation of ectopic lesions, and the environment is closely related to the pathogenesis of endometriosis and influence on pregnancy outcomes[15].

Among many immune factors, the imbalance of innate immunity of endometriosis is an important reason for the occurrence and development of the disease. STING is an important molecular protein related to innate immunity. As a DNA receptor, it can recognize cellular DNA released. Endogenous molecules released by endometriosis due to tissue damage or inflammatory effect, such as HMGB1 and IL-33, can activate this signaling pathway[21, 22]. The innate immune system can recognize many invaded pathogens. As a cytokine released by recognizing these microbial molecules, type I interferon plays an important role in eliminating pathogens. The release of type I interferon mainly depends on TBK1 and downstream transcription regulator IRF3. In addition, transmembrane protein STING is an important cellular DNA receptor, which can recognize intracellular DNA then activate TBK1 / IRF3 signaling pathway released type I interferon. In our study, the expression of STING in eutopic endometrial tissue of endometriosis, which called a "benign cancer", was decreased, but the proliferation, invasion and migration of endometrial stromal cells increased. These made us suspect that STING may have the same anti-tumor effect in endometriosis, which can be used to prevent ectopic lesions formation, and we found its signal transduction pathway in endometrial stromal cells had changed. Moreover, exogenous IFNB1 reduced the invasion and migration of stromal cells, which also showed that STING may play the same role in tumor immunotherapy, and provide new ideas for treatments of endometriosis. 
The advantages of our study, firstly, we found that the expression of STING was decreased compared with controls, and mainly expressed in eutopic endometrial stroma of endometriosis, yet expressed in glandular epithelium of ectopic endometrium. Secondly, we first showed that STING had effects on function of endometrial stromal cells, and its low expression promoted the invasion and migration of stromal cells. Thirdly, we first explained that STING-IRF-IFNb1 signaling pathway played roles in endometrial stromal cells; However, our research still has some shortcomings. First, STING, as an endoplasmic reticulum anchoring protein, can recognize DNA released and act as a DNA receptor. Therefore, we need to study the relationship between STING and endoplasmic reticulum stress, DNA damage repair, and the impact of these relationships on the occurrence and development of endometriosis. Second, the experiments in this chapter were in vitro experiments, which still need to be in vivo experiments, in order to carry out the verification and deeper mechanism research.

In conclusion, STING expressed lower in eutopic endometrial tissue of endometriosis, and mainly expressed in stroma. Its low expression promoted the invasion and migration of endometrial stromal cells. STING/IRF3/IFNb1 signaling pathway plays an important role in endometrial stromal cells. The addition of exogenous IFNB1 can reversely reduce the invasion and migration of stromal cells, which may be explained the pathogenesis mechanism of endometriosis and provided new ideas of treatment methods.

\section{Conclusion}

In eutopic endometrium of endometriosis, STING expression was decreased and mainly in the stroma. Low expression of STING can promote the invasion and migration of endometrial stromal cells. It is further found that of STING/IRF3/IFNb1 signaling pathway in functions of HESC, and the addition of exogenous IFNB1 can reverse and reduce the invasion and migration of stromal cells.

\section{Abbreviations}

STING: stimulator of interferon genes; WB: western blot assay; HESC: human endometrial stromal cell; IHC: Immunohistochemistry

\section{Declarations}

\section{Ethics approval and consent to participate}

This research was approved by the Ethics Committee of the Reproductive Hospital Affiliated to Shandong University. Written informed consent was obtained from all participants before study enrollment.

\section{Consent for publication}

All authors have consented to the publication of this article. 
Availability of supporting data

All data generated through this study are included in this article

\section{Competing interests}

The authors declare no potential conflicts of interest with respect to the research, authorship, and/or publication of this article.

\section{Funding}

National Natural Science Foundation of China (82071617)

\section{Authors' contributions}

ZX drafted the manuscript and revised it critically for important intellectual content. ZX, HZ, CY, LZ, ML and $\mathrm{YW}$ contributed to the conception and design of the work or the acquisition, analysis and interpretation of the data. LY approved of final the version to be published, and agree to be accountable for all aspects of the work in ensuring that

questions related to the accuracy or integrity of any part of the work are appropriately investigated and resolved.

\section{Acknowledgements}

We would like to thank all the endometriosis patients for their participation during this study.

\section{References}

1. Zondervan, K.T., et al., Endometriosis. Nat Rev Dis Primers, 2018.

2. Shigesi, N., et al., The association between endometriosis and autoimmune diseases: a systematic review and meta-analysis. Human Reproduction Update, 2019. 25(4): p. 486-503.

3. Vallvé-Juanico, J., S. Houshdaran, and L.C. Giudice, The endometrial immune environment of women with endometriosis. Human Reproduction Update, 2019. 25(5): p. 565-592.

4. Patel, B.G., et al., Pathogenesis of endometriosis: Interaction between Endocrine and inflammatory pathways. Best Pract Res Clin Obstet Gynaecol, 2018.

5. Yi, K.W., et al., Endometriosis Is Associated with Adverse Pregnancy Outcomes: a National Population-Based Study. Reprod Sci, 2020.

6. Akira, S., S. Uematsu, and O. Takeuchi, Pathogen recognition and innate immunity. Cell, 2006.

7. Yao, Z., et al., Deciphering biomarkers of endometriosis by proteomic analysis of eutopic endometrium in infertile patients. J Gynecol Obstet Hum Reprod, 2020. 
8. Burdette, D.L., et al., STING is a direct innate immune sensor of cyclic di-GMP. Nature, 2011. 478(7370): p. 515-518.

9. Le Naour, J., et al., Trial watch: STING agonists in cancer therapy. Oncolmmunology, 2020. 9(1): p. 1777624.

10. Tlemsani, C., et al., SCLC-CellMiner: A Resource for Small Cell Lung Cancer Cell Line Genomics and Pharmacology Based on Genomic Signatures. Cell Rep, 2020.

11. Xu, N., et al., STING agonist promotes CAR T cell trafficking and persistence in breast cancer. J Exp Med, 2021.

12. Zhang, Y., et al., $\beta$-arrestin 2 as an activator of cGAS-STING signaling and target of viral immune evasion. Nat Commun, 2020.

13. Qu, H., et al., Epithelial Cells in Endometriosis and Adenomyosis Upregulate STING Expression. Reprod Sci, 2020.

14. Lessey, B.A., The pathologists are free to go, or are they? Fertil Steril, 2013.

15. Saraswat, L., et al., Pregnancy outcomes in women with endometriosis: a national record linkage study. BJOG, 2017.

16. Houshdaran, S., et al., Aberrant Endometrial DNA Methylome and Associated Gene Expression in Women with Endometriosis. Biol Reprod, 2016.

17. Tamaresis, J.S., et al., Molecular classification of endometriosis and disease stage using highdimensional genomic data. Endocrinology, 2014.

18. Ahn, S.H., et al., Immune-inflammation gene signatures in endometriosis patients. Fertil Steril, 2016.

19. Aghajanova, L., M.C. Velarde, and L.C. Giudice, Altered gene expression profiling in endometrium: evidence for progesterone resistance. Semin Reprod Med, 2010.

20. Bruner-Tran, K.L., et al., Medical management of endometriosis: emerging evidence linking inflammation to disease pathophysiology. Minerva Ginecol, 2013.

21. Zachariah, R., et al., Circulating cell-free DNA as a potential biomarker for minimal and mild endometriosis. Reproductive Biomedicine Online, 2009. 18(3): p. 407-411.

22. Traver, S., et al., Cell-free nucleic acids as non-invasive biomarkers of gynecological cancers, ovarian, endometrial and obstetric disorders and fetal aneuploidy. Human Reproduction Update, 2014(6): p. 905.

\section{Figures}


A

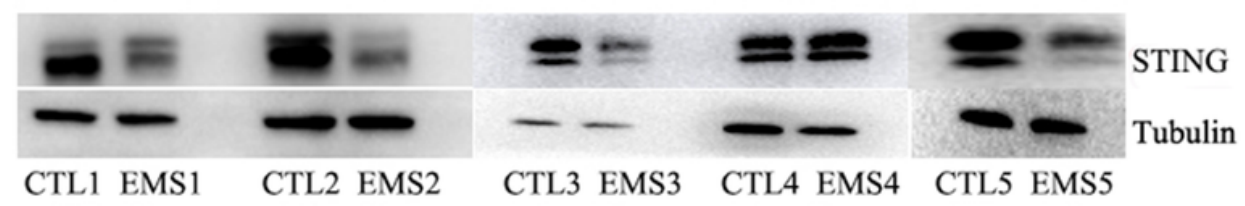

B

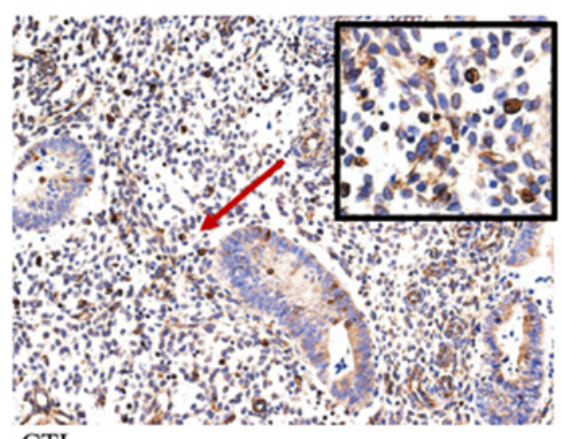

CTL
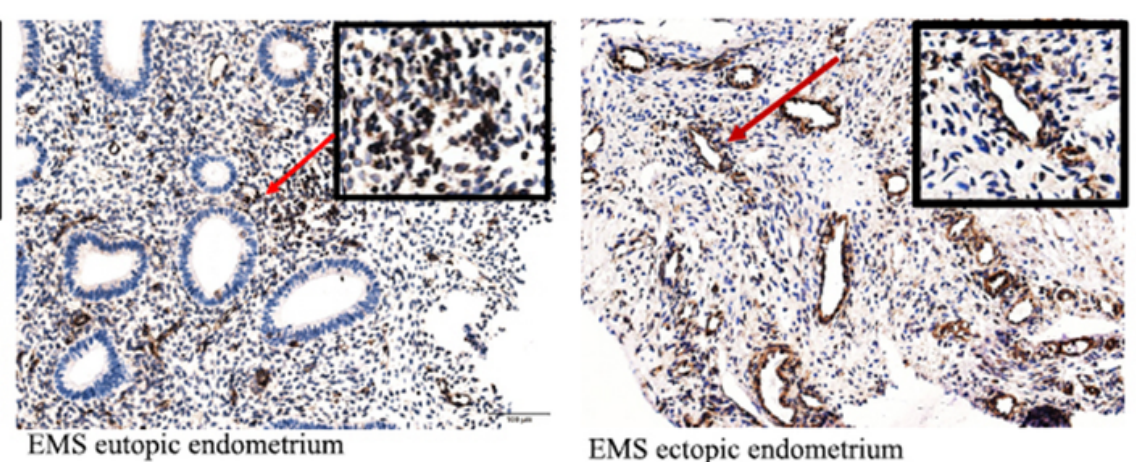

EMS ectopic endometrium

\section{Figure 1}

The expression of STING in eutopic endometrium of endometriosis (A) WB results showed that the expression of STING in eutopic endometrium of endometriosis was decreased compared to the controls, (B) IHC results showed that STING was significantly expressed in the stroma and glandular epithelium in control endometrium. The expression of STING was mainly in the stroma of eutopic endometrium of endometriosis was decreased, but expressed in glandular epithelium of ectopic endometrium. Each experiment was repeated three times 
A
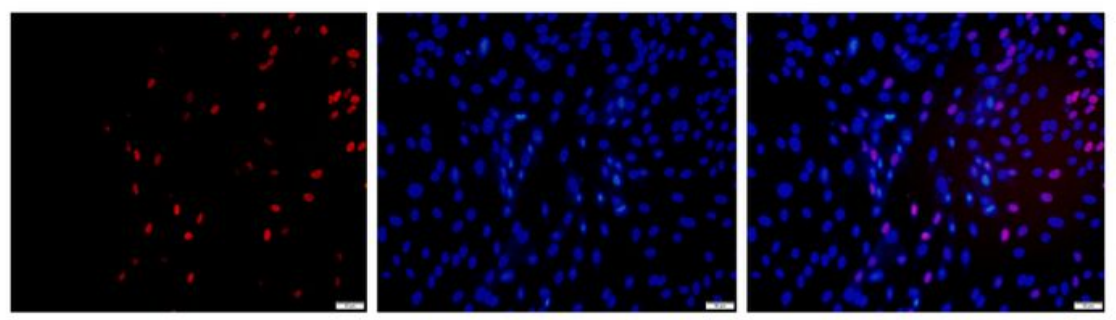

NC

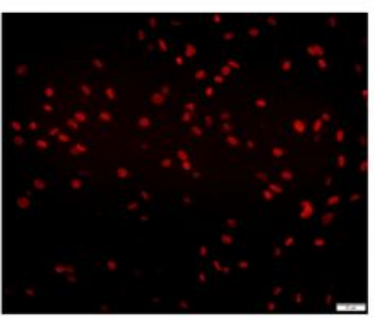

EdU

B
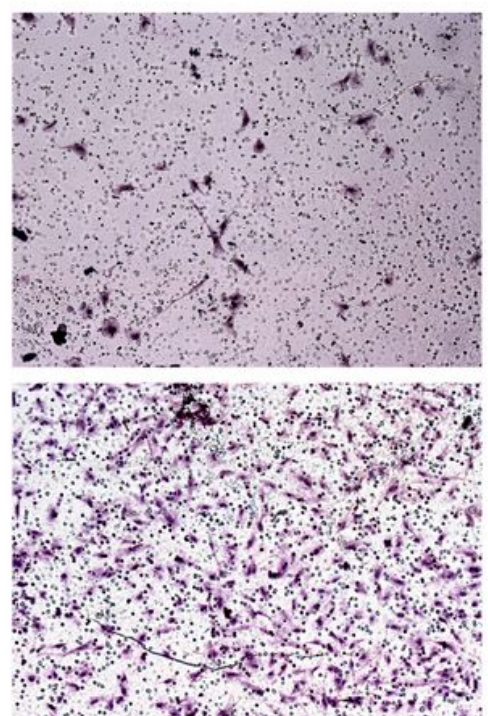

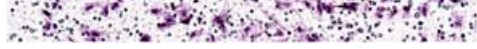

NC

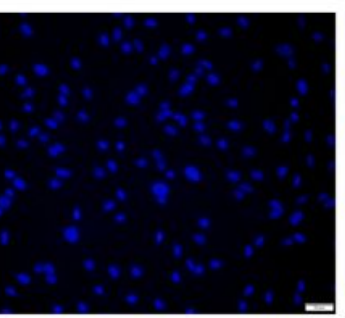

DNA
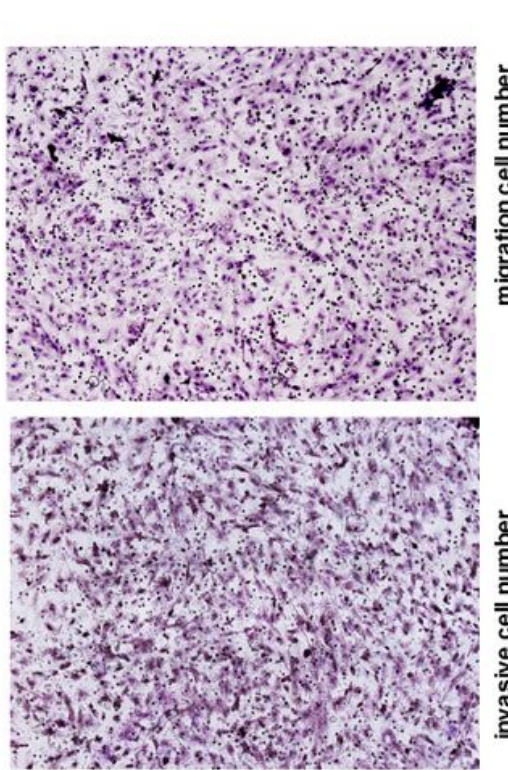

Si-STING

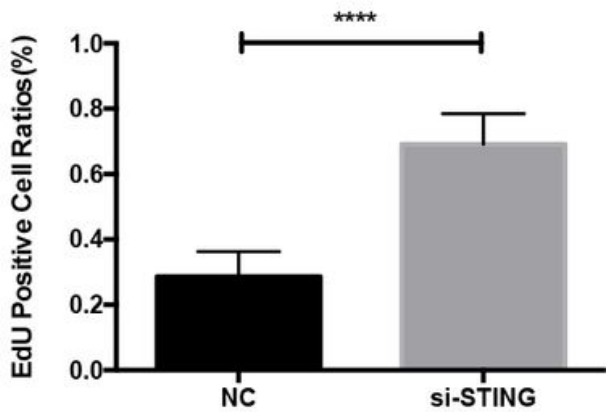

Si-STING
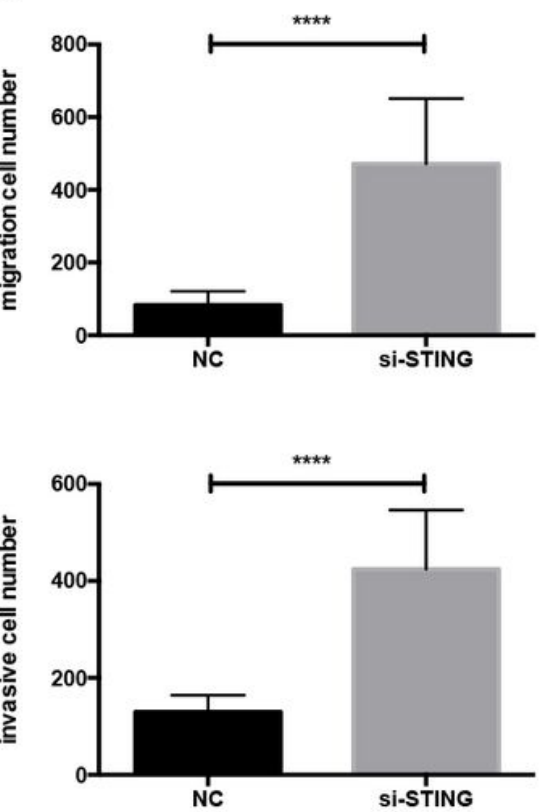

Figure 2

Effect of STING on the function of human endometrial stromal cells (HESC) (A) Edu results showed that low expression of STING can promote the proliferation level. According to EdU positive cells rate, STING knockdown (siSTING) in HESC compared to NC group $0.2866 \pm 0.01470$ vs. $0.6911 \pm 0.01796$, $\star \star \star \star P<0.0001$. (B) And Transwell results showed that low expression of sting could promote the invasion ( $130.0 \pm 6.296$ vs. $424.1 \pm 22.31, * \star \star \star P<0.0001)$ and migration ( $82.93 \pm 6.940$ vs. $82.93 \pm 6.940$, $\star \star \star \star \mathrm{P}<0.0001)$ of HESC when knocked down STING. Each experiment was repeated three times 
A

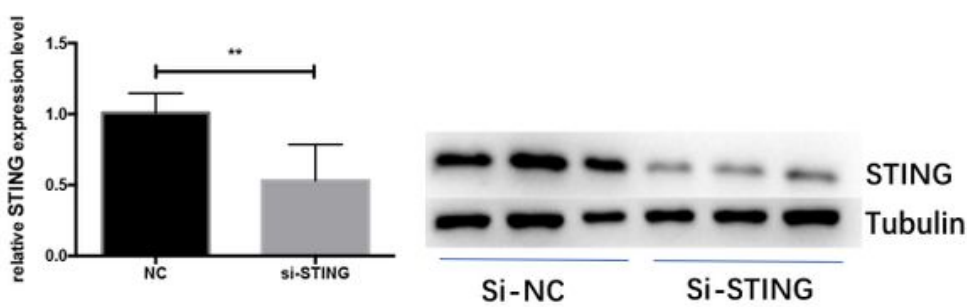

B
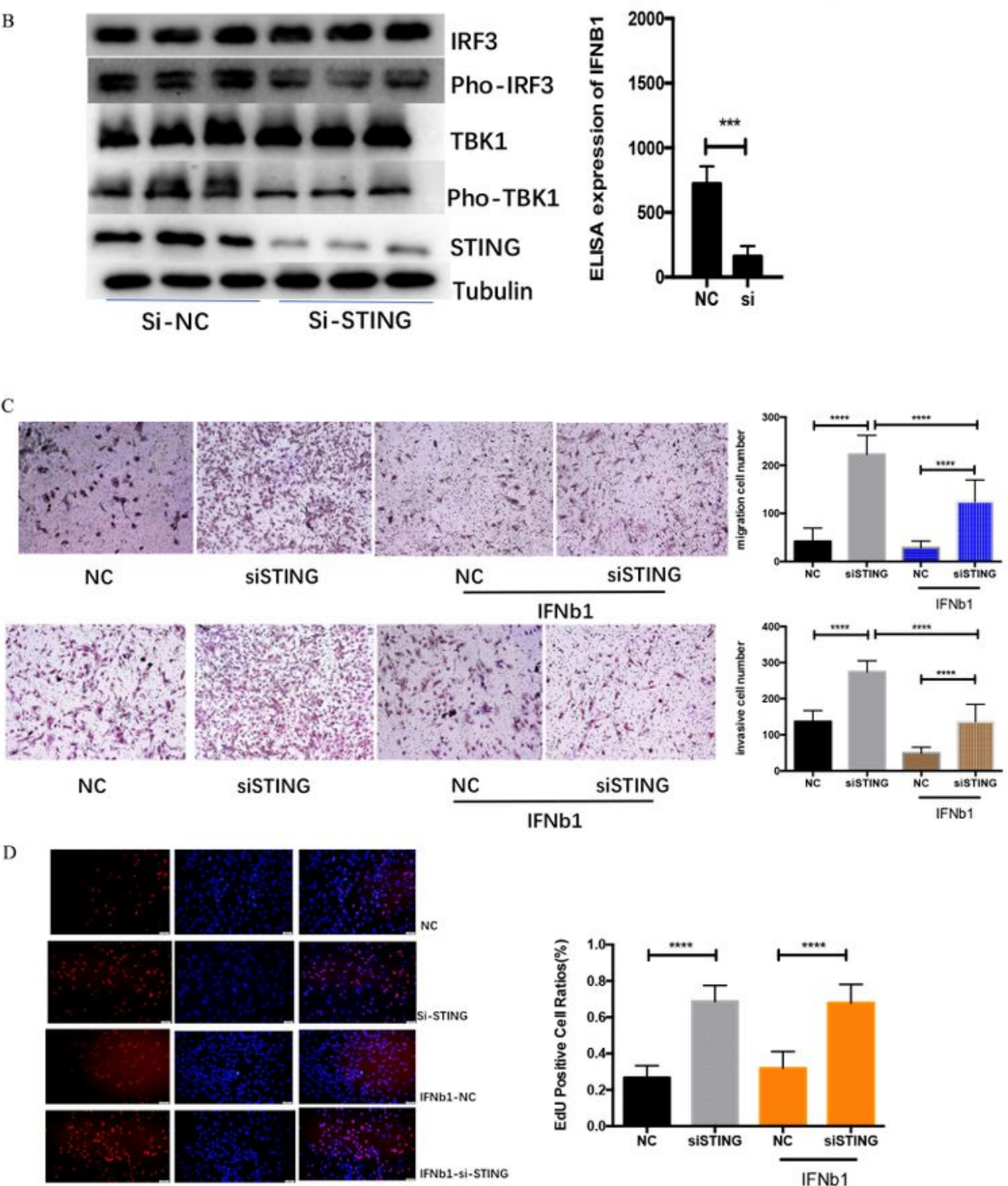

Figure 3

STING/IRF3/IFNb1 signaling pathway in HESC (A) knocked down STING (si-STING) in HESC cells, WB results showed that STING expression was significantly decreased. (B) and (C) WB results showed that expressions of phosphorylated IRF3 and TBK1 with knockdown STING were lower than that of total protein IRF3 and tbk1 in STING/IRF3/IFNb1 pathway in HESC. ELISA results showed that the expression of IFNb1 with knockdown STING was lower than NC group ( $\left.{ }^{*} P<0.05\right)$. (D) and (E) Transwell results 
showed that exogenous IFNB1 could reduce the invasion (IFNb1-NC and IFNb1-siSTING $274.7 \pm 7.767$ vs. $135.7 \pm 12.63, \star \star \star P<0.0001)$ and migration (IFNb1-NC and IFNb1-siSTING $28.53 \pm 3.625$ vs. $28.53 \pm$ 3.625 , $* \star * P<0.0001)$ of HESC with knockdown STING, but could not completely reverse the invasion (siSTING and IFNb-siSTING $222.9 \pm 10.16$ vs. $124.1 \pm 11.77$, $* \star * P<0.0001$ ) and migration (siSTING and IFNb-siSTING $49.13 \pm 4.241$ vs. $135.7 \pm 12.63, * \star \star * P<0.0001)$. EdU results showed that exogenous added IFNB1 did not decrease the proliferation of HESC with knockdown STING (siSTING and IFNb-siSTING $0.6874 \pm 0.02081,0.7187 \pm 0.02638, P=0.795)$. Each experiment was repeated three times

\section{Supplementary Files}

This is a list of supplementary files associated with this preprint. Click to download.

- floatimage1.png 